University of Nebraska - Lincoln

DigitalCommons@University of Nebraska - Lincoln

Norman R. Simon Papers

Research Papers in Physics and Astronomy

3-15-1983

\title{
The Light and Velocity Curves of Classical Cepheids: Theory Versus Observation
}

Norman R. Simon

University of Nebraska - Lincoln, nsimon@unl.edu

Cecil G. Davis

University of California

Follow this and additional works at: https://digitalcommons.unl.edu/physicssimon

Simon, Norman R. and Davis, Cecil G., "The Light and Velocity Curves of Classical Cepheids: Theory Versus Observation" (1983). Norman R. Simon Papers. 17.

https://digitalcommons.unl.edu/physicssimon/17

This Article is brought to you for free and open access by the Research Papers in Physics and Astronomy at DigitalCommons@University of Nebraska - Lincoln. It has been accepted for inclusion in Norman R. Simon Papers by an authorized administrator of DigitalCommons@University of Nebraska - Lincoln. 
THE ASTRophysical Journal, 266:787-793, 1983 March 15

(C) 1983. The American Astronomical Society. All rights reserved. Printed in U.S.A

\title{
THE LIGHT AND VELOCITY CURVES OF CLASSICAL CEPHEIDS: THEORY VERSUS OBSERVATION
}

\author{
NoRman R. Simon \\ Behlen Laboratory of Physics, University of Nebraska-Lincoln \\ AND \\ Cecil G. Davis \\ University of California, Los Alamos Scientific Laboratory \\ Received 1982 August 4; accepted 1982 September 8
}

\begin{abstract}
Fourier decomposition is employed to compare observed light and velocity curves of classical Cepheids with light and velocity curves generated by two sets of hydrodynamic models-the Carson-opacity models of Vemury and Stothers (VS) and a set of models constructed with Los Alamos opacities and dynamical zoning (LOS). In spite of the fact that a number of the theoretical light and (particularly) velocity curves appear to the eye to resemble the observations, the Fourier decompositions reveal severe shortcomings in both sets of calculations. The VS velocity curves are found superior to LOS in reproducing sharp resonance effects in the observations, but the VS light curves are not nearly so successful. With regard to the LOS calculations, resonance effects in both the light and velocity curves are generally much muted compared with their striking presence in the observational data. At the moment it is not clear if the difference between VS and LOS is an opacity effect. Finally, we examine various definitions of the light-radius phase lag in the theoretical models. It is suggested that a phase lag obtained from the Fourier decompositions will ultimately be the most useful for comparison with observation. When the phase lag is defined in this manner, the dynamically zoned LOS models produce the most plausible and consistent results.
\end{abstract}

Subject headings: stars: Cepheids - stars: interiors - stars: pulsation

\section{INTRODUCTION}

Since the advent of nonlinear pulsation codes 20 years ago, it has been possible to compare in some detail observed light and velocity curves with those generated by theoretical models. In the ensuing time many such comparisons have been made (see, e.g., Cox 1974), mostly qualitative, and have treated such properties of the variations as phase lags, asymmetries, amplitudes, and the presence of distinguishing features such as bumps, shoulders, standstills, etc. In several cases, theoretical light or velocity curves have been matched directly with data from individual stars (e.g., Christy 1966; Carson, Stothers, and Vemury 1981). One particularly fruitful, yet disturbing, instance of the comparison of theory and observation has involved the Hertzsprung progression among classical Cepheids. This work has given rise to a period-ratio anomaly and to the association of light curve features with a period resonance in the pulsation models. A review of this problem has been given by Cox (1980).

Up until recently, however, no systematic description has existed of the structure of observed light and velocity curves which would allow quantitative techniques to be applied to a large sample of pulsating stars. A step in this direction was made by Simon and Lee (1981), who Fourier analyzed the light curves of 57 classical Cepheids. It was found that a quantitative description of the progression of curve shape with period could be obtained in terms of combinations of the low-order Fourier coefficients. Subsequently, the same technique was applied to the light curves of RR Lyrae stars (Simon and Teays 1982) and to the velocity curves of classical Cepheids (Simon and Teays 1983). This method has also been used by Hodson, Cox, and King (1982) to treat the computed variations of BL Herculis models. However, a quantitative comparison with actual stars was not possible in this case because of a lack of suitable observational data.

In the present work we shall obtain nonlinear hydrodynamic models of the classical Cepheid pulsators and subject the light and velocity curves generated by these models to Fourier decomposition. We shall then be able to compare the observed and calculated variations by matching their respective Fourier coefficients. The results of this investigation will show that the theoretical models have some serious defects in spite of apparent qualitative agreement in "eyeball" comparisons between the observed light and velocity curves and their hydrodynamically modeled counterparts. 


\section{MODELS AND FOURIER DECOMPOSITION}

The theoretical models we shall employ come from two sources. The first set of models was constructed by Vemury and Stothers (1978). These four models are characterized by $M / M_{\odot}=7$ and $\log \left(L / L_{\odot}\right)=3.7$. Their properties are described in Table 1 , and their light and velocity curves illustrated in Figures 5 and 6 of the above reference. The Vemury-Stothers (VS) models are particularly interesting because they were calculated with the controversial Carson opacities (Carson 1976).

The second set of models was produced for the present investigation using the dynamically zoned Los Alamos pulsation code described by Adams, Castor, and Davis (1980). Linear nonadiabatic (LNA) counterparts of these models were also constructed, both versions with Los Alamos opacities and the King IVa composition. Table 1 lists some properties of the Los Alamos (LOS) models, including the nonlinear fundamental period $P_{0}(\mathrm{NL})$, the linear fundamental period $P_{0}(\mathrm{LNA})$, and the linear period ratio of second overtone to fundamental $P_{2} / P_{0}$ (LNA).

The light and velocity curves for our 18 theoretical models (4 from Vemury-Stothers and 14 from the present work) were subjected to Fourier analysis in the manner described by Simon and Lee (1981) and Simon and Teays (1983). Before the analysis, the luminosity variations were always expressed in terms of $M_{\text {bol }}$, and the velocities in "observational coordinates," i.e., positive velocity indicating a contraction of the star. In this form, the theoretical results are directly comparable to observations.

The theoretical curves produced with the Los Alamos code were smoother than the VS results, but in all cases the Fourier decompositions were good, meeting or exceeding the criteria described by Simon and Lee (1981). This means that we consider the lower order coefficients to be well determined, thus truly reflecting the structural characteristics of the modeled oscillations. The Fourier fits that emerged were generally of eighth order, but in a few cases fourth-order versions were deemed sufficient.

\section{THEORY VERSUS OBSERVATION}

The Fourier fits to $M_{\text {bol }}$ have the form

$$
A_{0}+A_{i} \cos \left(i \omega t+\phi_{i}\right)
$$

while for the velocities, the fitting scheme is

$$
A_{0}-A_{i} \sin \left(i \omega t+\phi_{i}\right)
$$

In treating the observed light variations Simon and Lee (1981) considered the quantities $\phi_{21}=\phi_{2}-2 \phi_{1}, R_{21}$ $=A_{2} / A_{1}$, and $\phi_{31}=\phi_{3}-3 \phi_{1}$. For the case of the observed velocities, only $\phi_{21}$ and $R_{21}$ were considered (Simon and Teays 1983). In the present investigation we shall compare theory and observation in terms of the
TABLE 1

\begin{tabular}{|c|c|c|c|c|c|}
\hline$M / M_{\odot}$ & $L / L_{\odot}$ & $T_{e}$ & $P_{0}(\mathrm{NL})$ & $P_{0}(\mathrm{LNA})$ & CNA) \\
\hline 4.00 & 1970 & 6000 & 5.30 & 5.32 & 0.553 \\
\hline 4.02 . & 2610 & 5900 & 7.18 & 7.17 & 0.535 \\
\hline $4.00 \ldots \ldots$ & 2950 & 5800 & 8.38 & 8.47 & 0.518 \\
\hline 4.26 . & 3080 & 5800 & 8.43 & 8.45 & 0.527 \\
\hline $4.40 \ldots$ & 3610 & 5750 & 9.66 & 9.75 & 0.522 \\
\hline $4.00 \ldots$ & 3240 & 5700 & 9.70 & 9.84 & 0.515 \\
\hline $3.50 \ldots \ldots$ & 2880 & 5700 & 9.72 & 9.76 & 0.503 \\
\hline $4.80 \ldots \ldots$ & 4390 & 5650 & 11.62 & 11.56 & 0.511 \\
\hline $3.90 \ldots \ldots$ & 3140 & 5400 & 11.62 & 11.86 & 0.495 \\
\hline $4.98 \ldots \ldots$ & 5440 & 5600 & 13.75 & 14.10 & 0.501 \\
\hline $5.00 \ldots \ldots$ & 5230 & 5500 & 14.40 & 14.52 & 0.501 \\
\hline $4.50 \ldots \ldots$ & 5230 & 5500 & 15.75 & 15.76 & 0.487 \\
\hline $4.30 \ldots \ldots$ & 5230 & 5500 & 16.00 & 16.28 & 0.480 \\
\hline $4.00 \ldots \ldots$ & 5230 & 5500 & 17.20 & 17.16 & 0.467 \\
\hline
\end{tabular}

PROPERTIES OF THE LOS MODELS

best determined of these quantities-namely, $\phi_{21}$ and $R_{21}$ for the light and $\phi_{21}$ for the velocity. Thus we are dealing with observed properties of the variations as given for the light in Figures 1 and 2 of Simon and Lee ( $\phi_{21}$ and $R_{21}$ versus period) and for the velocity in Figure 1 of Simon and Teays (1983) ( $\phi_{21}$ versus period).

Figure 1 of the present work displays a plot of $\phi_{21}$ versus period for the light variations of the theoretical

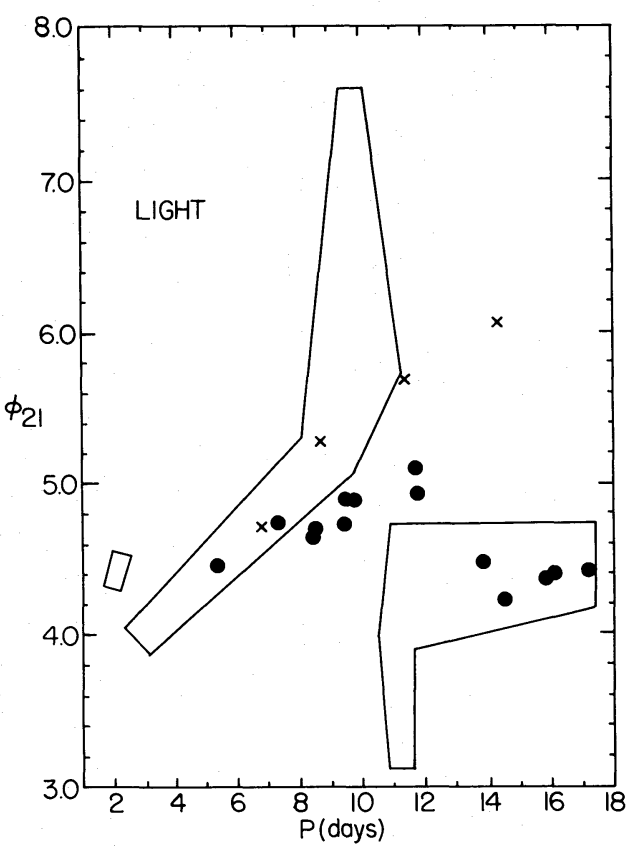

FIG. 1. - The quantity $\phi_{21}=\phi_{2}-2 \phi_{1}$ vs. period for the light variations of the theoretical models. Closed circles, LOS models; crosses, VS models. Envelopes have been sketched in to represent the observational data. 


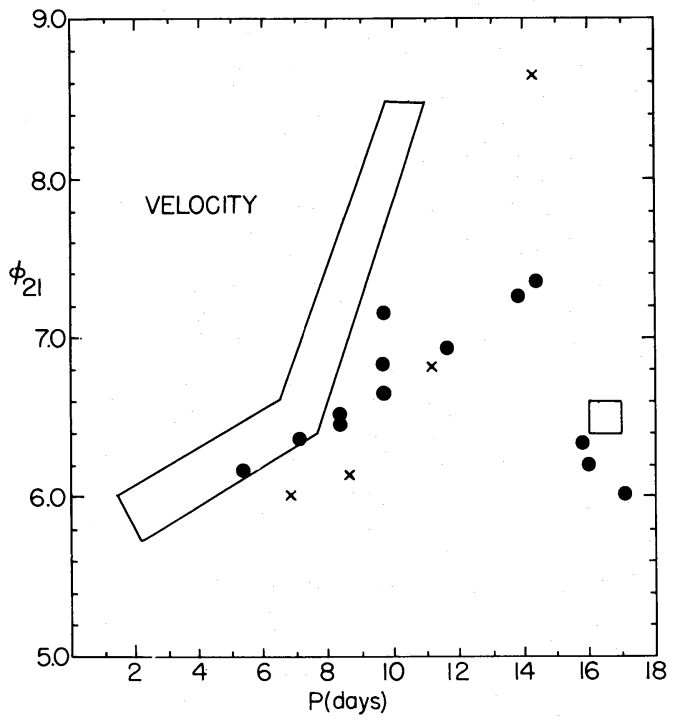

FIG. 2. $-\phi_{21}$ vs. period for the theoretical velocity variations. Notation as in Fig. 1.

models. Closed circles represent the LOS models, while the crosses denote the models of VS. Boundaries of the regions occupied by the observational points have been sketched in for comparison. The sharp break in the observations at 10 days is believed to be due to the period resonance $P_{2} / P_{0}=0.5$ (Simon and Schmidt 1976). One notices immediately the flatness in the distribution of LOS points across 10 days as compared with the observations. The VS points, on the contrary, do show a rise, but with the wrong slope and with a peak at too long a period.

In Figure 2 we plot $\phi_{21}$ versus period for the velocity variations. Once more the resonance appears clearly in the observations. The LOS points are seen to rise with much too shallow a slope and to peak at too long a period. On the other hand, the VS points resemble the observational envelope fairly well, except that the whole distribution of crosses is shifted toward longer periods.

At this point, it is instructive to note that if the resonance theory of Simon and Schmidt is correct, it is the period ratio $P_{2} / P_{0}$ rather than the fundamental period $P_{0}$ that is the principal mediator of curve shape. With this in mind we replot $\phi_{21}$ for both light (Fig. 3) and velocity (Fig. 4) with $P_{2} / P_{0}$ as abscissa. Although the observations cannot accurately be entered on such a diagram (since $P_{2} / P_{0}$ is not directly measurable), we have, nonetheless, plotted points representing our 18 hydrodynamic models. Similar diagrams have been made previously by Hodson, Cox, and King (1982) for models representing the BL Herculis stars.

Figure 3 again emphasizes the flatness of the LOS model points across the resonance, compared with the rather slow rise for the models of VS. A barely discern-

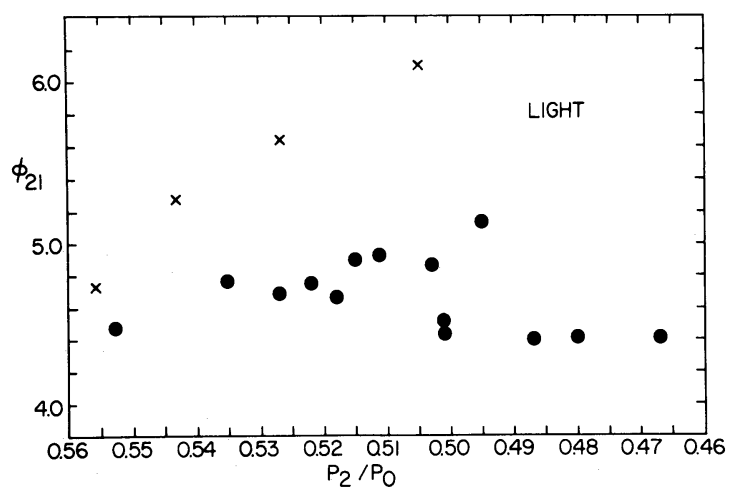

FIG. 3. $-\phi_{21}$ vs. $P_{2} / P_{0}$ for the light variations. Closed circles, LOS models; crosses, VS models.

ible peak in the LOS data appears near $P_{2} / P_{0}=0.5$, with a subsequent fall for smaller period ratios. The VS points also appear to peak near the resonance; but unfortunately, no models are available with $P_{2} / P_{0}<0.5$.

Turning to the velocity analysis in Figure $4, \phi_{21}$ for the LOS models exhibits a definite peak at the resonance center. However, the maximum attained is very modest compared with that displayed by the VS points, which again appear to peak at $P_{2} / P_{0}=0.5$ and whose sharp slope is very reminiscent of the observations. Taken together, Figures 2 and 4 suggest that if VS-type models could be constructed in proper relation to the resonance (i.e., $P_{2} / P_{0}=0.5$ at 10 days), their Fourier coefficients would match those of the observed velocity curves quite well. However, the VS light variations are decidedly different from the observations, while the LOS models do not provide a good fit in either velocity or light.

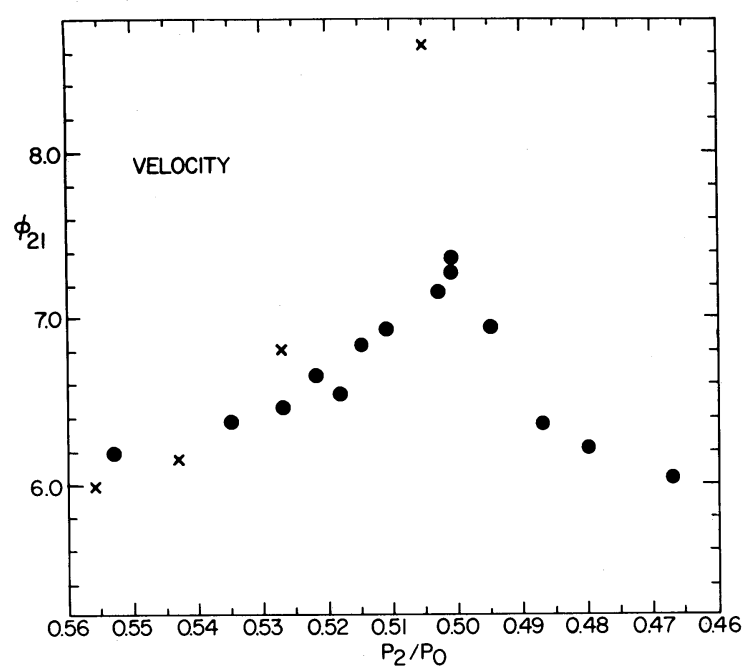

FIG. 4. $-\phi_{21}$ vs. $P_{2} / P_{0}$ for the velocity variations. Notation as in Fig. 3. 


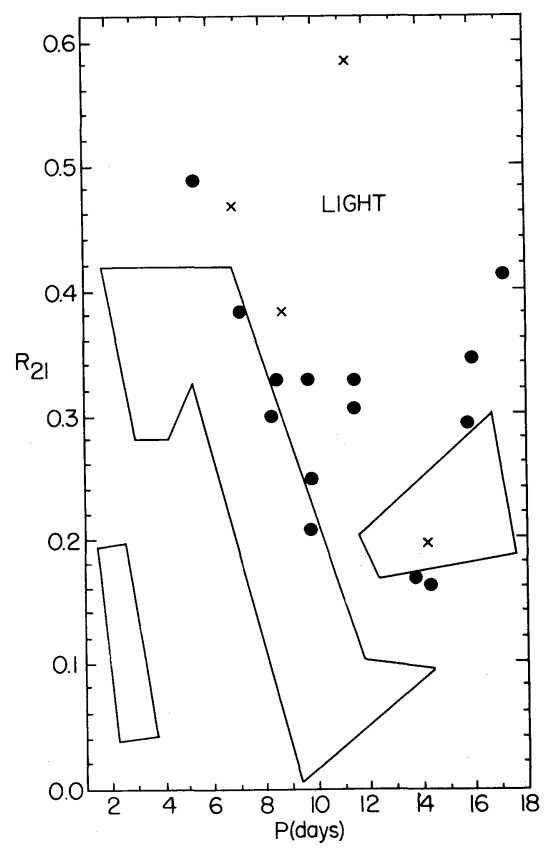

FIG. 5. $-R_{21}=A_{2} / A_{1}$ vs. period for the theoretical light variations. Notation as in Fig. 1.

In Figure 5 we present a plot of $R_{21}$ versus period for the light. Once more the observational envelopes have been blocked out by solid lines. One notes that the theoretical points again seem to be shifted to the right with respect to the observations. However, if a plot is made of $R_{21}$ versus period ratio $P_{2} / P_{0}$ (see Fig. 6), both the LOS and VS models produce an $R_{21}$ which bottoms out at the resonance. The LOS points then rise again at smaller period ratios for which no VS models are available. The VS cross at $P_{0} \approx 11$ days, $P_{2} / P_{0} \approx 0.53$ attains a value of $R_{21}$ considerably above that of any other point, calculated or observed. The increase in $R_{21}$ represented by this model may parallel the observed behavior at about 7 days (allowing for the fact that if the VS

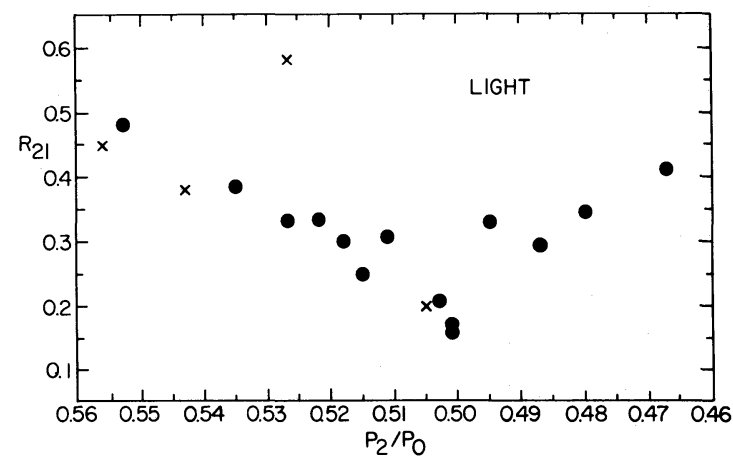

FIG. 6. $-R_{21}$ vs. $P_{2} / P_{0}$ for the theoretical light variations. Notation as in Fig. 3. models were constructed "on resonance," the period ratio $P_{2} / P_{0} \approx 0.53$ would appear at $P_{0} \approx 7$ days), but there are too few models to verify this. On the other hand, the actual value of $R_{21}$ for the 11 day model clearly seems too high. In summary, we conclude that both the LOS and VS models crudely duplicate the main feature of the observed run of $R_{21}$ (i.e., the minimum at $P_{2} / P_{0} \approx 0.5$ ), with the LOS points perhaps slightly preferable.

\section{THE PHASE SHIFTS}

The phase lag in Cepheid pulsations between minimum radius and maximum luminosity has been an important test of theoretical models and thus, widely discussed in the literature. It is well known that, crudely speaking, the light maximum occurs not at the zero velocity phase corresponding to minimum radius but rather a quarter of a cycle later when the star attains its maximum velocity of expansion. Thus if we form the quantity $\Delta \phi=\omega t_{L}-\omega t_{V}$, where $\omega$ is the angular frequency of oscillation and $t_{L}$ and $t_{V}$ represent the time of maximum light and the time of maximum expansion velocity, respectively, the canonical value of $\Delta \phi$ is zero.

There exist in the literature a number of ways of defining and obtaining $\Delta \phi$. In the first place one can measure the quantity directly from the light and velocity curves, either observed or theoretical. Second, one can calculate the phase shift $(\Delta \phi)_{\text {LNA }}$, which comes from linear models. Finally, a third phase shift may be obtained by Fourier decomposition. This is the phase shift $(\Delta \phi)_{1}$ between the linear terms emerging from the Fourier analysis of the light and velocity curves. If the Fourier fits are as given by equations (1) and (2), we find

$$
(\Delta \phi)_{1}=\phi_{1}(\text { velocity })-\phi_{1}(\text { magnitude })+\frac{\pi}{2} .
$$

Since the papers of Castor $(1968,1971)$ and the success of LNA models in crudely reproducing the canonical phase shift, it has generally been accepted that the phase lag is a phenomenon which already appears in the linear theory, with the nonlinear terms supplying what is essentially "fine-tuning." To the extent that this is the case we ought to have $(\Delta \phi)_{\mathrm{LNA}} \approx \Delta \phi$. Furthermore, it was argued by Simon (1979a) that to the extent that the Fourier decompositions are dominated by firstorder terms, it should be true that $(\Delta \phi)_{1} \approx(\Delta \phi)_{\mathrm{LNA}}$. Indeed, it would not be surprising if the three quantities $\Delta \phi,(\Delta \phi)_{\mathrm{LNA}}$, and $(\Delta \phi)_{1}$ were approximately equal or at least related to one another in some systematic fashion.

In Figure 7 we plot against one another the phase shift $\Delta \phi$ read from the light and velocity curves of the hydrodynamic models and the phase shift $(\Delta \phi)_{\mathrm{LNA}}$ from linear theory. We have used here only the LOS models 


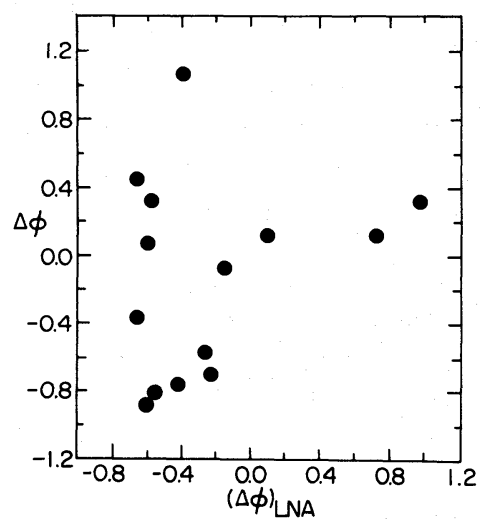

FIG. 7. - Raw phase shift $\Delta \phi$ vs. the linear phase shift $(\Delta \phi)_{\text {LNA }}$ for the LOS models.

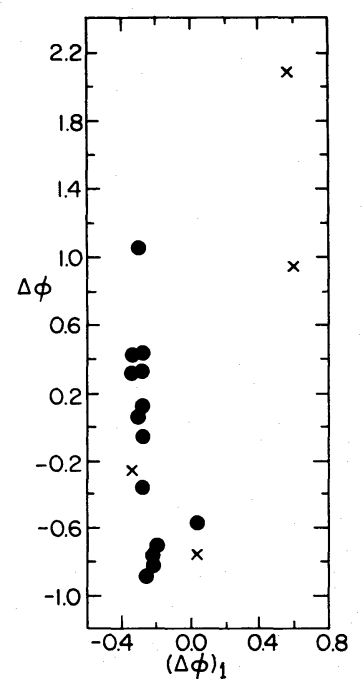

FIG. 8. - Raw phase shift $\Delta \phi$ vs. first-order nonlinear phase shift $(\Delta \phi)_{1}$. Notation as in Fig. 3.

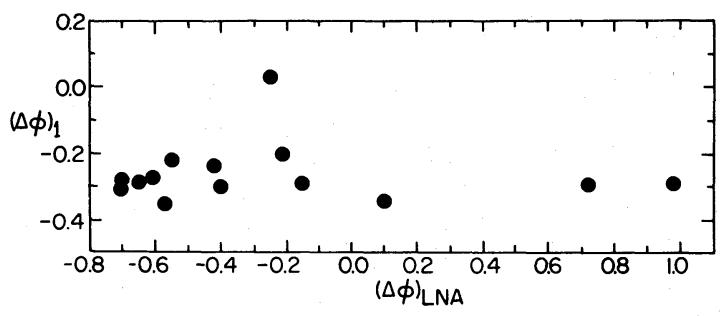

FIG. 9. - $(\Delta \phi)_{1}$ vs. $(\Delta \phi)_{\mathrm{LNA}}$ for the LOS models

since LNA results were not available in the case of VS. The points in Figure 7 show large scatter. While there may exist a weak trend with positive slope, it would clearly be very difficult to predict the phase shift $\Delta \phi$ of a given hydrodynamic model from the phase $\operatorname{shift}(\Delta \phi)_{\mathrm{LNA}}$ of its linear counterpart.
Figure 8 presents a plot of $\Delta \phi$ versus $(\Delta \phi)_{1}$, the latter determined by Fourier analysis. Since both quantities come from the hydrodynamic curves, the VS models have been included. The most striking property of Figure 8 is that the LOS models yield values of $(\Delta \phi)_{1}$ which fall in an extremely narrow range, something which is not true of the VS models. For neither set of models is $(\Delta \phi)_{1}$ a useful predictor of $\Delta \phi$.

Finally, we give in Figure 9 a graph of $(\Delta \phi)_{1}$ versus $(\Delta \phi)_{\mathrm{LNA}}$, again for the LOS models only. Once more we remark the strong homogeneity in $(\Delta \phi)_{1}$ centered about the value $(\Delta \phi)_{1} \approx-0.3$. We shall return to this property later.

\section{FOURIER ANALYSIS VERSUS THE EYE}

A number of authors have constructed hydrodynamic models which appear to the eye to reproduce the Hertzsprung or "bump" sequence for middle-period Cepheids. Among these are Stobie (1969, undermassive models), Vemury and Stothers (1978, Carson-opacity models), and Hodson and Cox (1980, He-enriched models). The first and last of these not only look appropriate to the eye, but also satisfy the requirements of the resonance hypothesis of Simon and Schmidt (1976). The LOS calculations from the present work resemble the Stobie models in being undermassive and also produce bumps in approximately the proper places as indicated by $P_{2} / P_{0}$.

However, when one attempts to match theory and observation via Fourier decomposition, it becomes clear that the eyeball comparisons are often quite unreliable. This is well illustrated by the sequence of velocity curves for the VS models given in Figure 6 of Vemury and Stothers (1978). These authors claim (with justification if one relies on the eye) that the bump shifts from the descending to the ascending branch as one passes from the 11.2 day model to the 14.3 day model. However, the Fourier decompositions in Figures 2, 4, and 6 show that, in underlying structure, the 14.3 day model resembles not a longer period star like TT Aql, but rather a 10 day, low-amplitude Cepheid like $\zeta$ Gem. This is consistent with the period ratio of the 14.3 day model, namely, $P_{2} / P_{0}=0.505$.

The above discussion indicates that eyeball monitoring of the absence or presence of bumps and of their locations on light or velocity curves leaves much to be desired as a description of the Hertzsprung sequence. As Vemury and Stothers (1978) have pointed out, a number of models which should not have shown bumps according to the resonance hypothesis in fact did show them, while other models with periods appropriate for bumps along the Hertzsprung sequence did not seem to exhibit such features. A similar confusion may be found in the observations themselves. Consider, for example, the five 
Cepheids VV Cas, BP Cas, RS Cas, RR Lac, and U Sgr with periods between 6.2 and 6.7 days, whose light curves are given by Mitchell et al. (1964). These light curves show bumps on their descending branches which range from strong (U Sgr) to weak (RS Cas) to virtually nonexistent (RR Lac). On the other hand, all of these stars lie very close together on the $\phi_{21}$ and $R_{21}$ diagrams of Simon and Lee (1981). Even the small-amplitude variable V496 Aql (Mitchell et al. 1964) is indistinguishable from its neighbors in the $\phi_{21}$ diagram though its light curve appears to the eye quite distinct.

While it is clear that the Fourier decompositions disallow the claim of Vemury and Stothers that Carsonopacity models have reproduced the bump sequence at correct periods with evolutionary masses, they also invalidate the contention of Hodson and Cox (1980) that the VS features are "surface disturbances" not connected with the Hertzsprung progression. On the contrary, Figures 2 and 4 indicate that the VS models have much to recommend them. In particular, it is difficult to believe that the sharp rise of $\phi_{21}$ and the fall of $R_{21}$ at the resonance sould be fortuitous.

\section{DISCUSSION}

The VS and LOS calculations display some significantly different properties. A major advantage of the VS models in reproducing the observations consists in the relatively strong resonance effects shown by these models, particularly in the velocity curves. For the LOS models, on the other hand, the resonance is weak in velocity and virtually nil in the light. While it is tempting to attribute this difference to the opacities, it is necessary to realize that not only were the two sets of models produced by different nonlinear codes, but the LOS models had a mass-to-light ratio only $60 \%$ that of the VS models at similar luminosity.

It would be important to pin down the source of the differing resonance response in the two sets of calculations not only for the "bump problem" itself but because resonance effects have been invoked by a number of authors to explain properties of pulsating stars in many regions of the H-R diagram (e.g., Simon 1979b; Borkowski 1980; Fitch 1980). Clearly, if certain types of models tend to accentuate resonance effects while others tend to suppress these effects, this characteristic could play an important role in the understanding of stellar pulsations.

Another problem touched in this investigation involves the phase shift between light and velocity. The Los Alamos LNA models produce a variety of phase shifts on both sides of (and in some cases straying quite far from) the canonical value $\Delta \phi=0$. Similar results have been noted quite generally among LNA models in the literature. According to Castor (1968), it must be nonlinear effects that are responsible for pulling the phase shifts close to the canonical value. However, if one examines the raw nonlinear phase shifts $\Delta \phi$, it is found from the present work that they are, if anything, more scattered than the LNA values for both the LOS and VS models. In the LOS calculations, on the other hand, the quantity $(\Delta \phi)_{1}$ exhibits a narrow range of values, consistent with the idea of ordering by nonlinear corrections.

We might understand the above result as follows. The LOS velocity and (particularly) light curves still contain some numerical noise in spite of efforts at smoothing. This noise is superposed on the basic physical structure of the variations and thus appears principally in the higher order coefficients in a Fourier decomposition. The quantity $(\Delta \phi)_{1}$, involving only the leading coefficients, is therefore able to demonstrate the nonlinear ordering, while the raw phase shift $\Delta \phi$, constructed from all the coefficients, is distorted by numerical noise. In the VS models, which lack dynamical zoning, some of the light curves are fairly smooth, while others are so noisy that even the lower order coefficients, and thus $(\Delta \phi)_{1}$, suffer distortion. In particular, one sees in Figure 8 that two of the VS models show values of $(\Delta \phi)_{1}$ quite compatible with the LOS models. Comparing Figure 5 of Vemury and Stothers (1978) it turns out that these two models $(P=6.85$ and $P=8.72)$ have relatively smooth light curves, whereas the other two models $(P=$ 11.2 and $P=14.3$ ) display much greater noise. The latter models, in turn, show values of $(\Delta \phi)_{1}$ which fall far from the norm.

Finally, it remains to inquire why the preferred value of $(\Delta \phi)_{1}$ emerging from the present models is $\sim-0.3$, rather than the canonical $\Delta \phi \sim 0$. While we cannot answer this question at the present time, it should be noted that observational values of $(\Delta \phi)_{1}$ are not yet determined for classical Cepheids but should be in the near future when simultaneous light and velocity curves become available. It is not precluded that the value -0.3 will actually emerge from the observations. In any event, it seems that so long as both observed and theoretical data remain imperfect in detail, the quantity $(\Delta \phi)_{1}$, rather than $\Delta \phi$, will be the more useful comparative measure of phase shift.

We are grateful to S. K. Vemury and R. Stothers for kindly supplying theoretical data and for informative discussions during the visit of one of us (N. R. S.) to the Institute for Space Studies in 1980. We would also like to thank T. J. Teays and D. Cooke for aiding in some of the calculations reported in this article, and T. Aikawa for an interesting discussion concerning the phase shifts. In addition, one of us (N. R. S) is pleased to acknowledge support from the National Science Foundation under grant AST-8105064. 


\section{REFERENCES}

Adams, T. F., Castor, J. I., and Davis, C. G. 1980, in Current Problems in Stellar Pulsation Instabilities, ed. D. Fischel, J. R. Lesh, and W. M. Sparks (NASA TM 80625), p. 175.

Borkowski, K. J. 1980, Space Sci. Rev., 27, 511.

Carson, T. R. 1976, Ann. Rev. Astr. Ap., 14, 95

Carson, T. R., Stothers, R., and Vemury, S. K. 1981, Ap. J., 244, 230.

Castor, J. I. 1968, Ap. J., 154, 793. 1971, Ap. J., 166, 109.

Christy, R. F. 1966, Ap. J., 144, 108

Cox, A. N. 1980, Ann. Rev. Astr. Ap., 18, 15

Cox, J. P. 1974, Rept. Progr. Phys., 37, 563.

Fitch, W. 1980, in Nonradial and Nonlinear Stellar Pulsation, ed H. A. Hill (Berlin: Springer-Verlag), p. 7.
Hodson, S. W., and Cox, A. N. 1980, in Nonradial and Nonlinear Stellar Pulsation, ed. H. A. Hill (Berlin: Springer-Verlag), p. 34. Hodson, S. W., Cox, A. N., and King, D. S. 1982, Ap. J., 253, 260. Mitchell, R. I., Iriarte, B., Steinmetz, D., and Johnson, H. L. 1964, Bol. Obs. Tonantzintla y Tacubaya, 3, 153.

Simon, N. R. $1979 a$, Astr. Ap., 74, 30 . $1979 b$, Astr. Ap. 75, 140

Simon, N. R., and Lee, A. S. 1981, Ap. J., 248, 291.

Simon, N. R., and Schmidt, E. G. 1976, Ap. J., 205, 162

Simon, N. R., and Teays, T. J. 1982, Ap. J., 261, 586. 1983, Ap. J., 265, 997.

Stobie, R. S. 1969, M.N.R.A.S., 144, 485.

Vemury, S. K., and Stothers, R. 1978, Ap. J., 225, 939 (VS).

C. G. DAvis: University of California, Los Alamos Scientific Laboratory, Box 1663, MS 240, Los Alamos, NM 87545

N. R. SimoN: Behlen Laboratory of Physics, University of Nebraska, Lincoln, NE 68588 